\title{
Avaliação Econômica dos Benefícios dos Macronutrientes em Diferentes Povoamentos de Bracatinga
}

\author{
Andressa Ribeiro $^{1}$, Sebastião do Amaral Machado² \\ ${ }^{1}$ Mestra em Engenharia Florestal, Universidade Federal do Paraná - UFPR, Curitiba/PR, Brasil \\ ${ }^{2}$ Departamento de Ciências Florestais, Universidade Federal do Paraná - UFPR, Curitiba/PR, Brasil
}

\begin{abstract}
RESUMO
A bracatinga é uma espécie nativa, com papel importante na economia de pequenos proprietários. Dessa forma, o presente trabalho foi desenvolvido com o intuito de quantificar o conteúdo de macronutrientes presentes nos diferentes componentes da biomassa aérea de bracatingais nativos e, posteriormente, valorar economicamente os resíduos florestais mantidos na área após a exploração. Os dados foram coletados em 304 parcelas temporárias, com diferentes idades, em povoamentos de bracatinga. Em ordem decrescente, os valores de macronutrientes encontrados na parte aérea dos bracatingais seguiram a sequência: $\mathrm{N}>\mathrm{Ca}>\mathrm{K}>\mathrm{Mg}>\mathrm{P}$. Após a exploração da lenha, restaram como remanescentes nas áreas os resíduos contendo uma média geral de $32 \%$ de $\mathrm{Ca}, 26 \%$ de $\mathrm{Mg}, 39 \%$ de N, $34 \%$ de $\mathrm{P}$ e $19 \%$ de $\mathrm{K}$, com um equivalente monetário médio de fertilizantes (em reais.ha ${ }^{-1}$ ) variando de 303,56 a 1.140,78, dependendo do local e da classificação de sítio da propriedade.
\end{abstract}

Palavras-chave: modelos de povoamento, resíduos florestais, fertilização.

\section{Economic Assessment of the Benefits of Macronutrients in Different Stand-levels of Bracatinga}

\begin{abstract}
Bracatinga (Mimosa Scabrella) is a native tree species with an important role in the economy of farmers. Thus, the present research was developed in order to quantify the contents of macronutrients present in the different compartments of biomass of native Bracatinga stands, and later, determine the economic valuation of the forest residues retained in the area after harvest. Data was collected from 304 temporary sample plots of Bracatinga stands with different ages. In decreasing order, the estimated values of macronutrients found in the above ground biomass of Bracatinga stands followed the sequence: $\mathrm{N}>\mathrm{Ca}>\mathrm{K}>\mathrm{Mg}>\mathrm{P}$. After firewood exploration in Bracatinga stands, $25 \% \mathrm{Ca}, 32 \% \mathrm{Mg}, 37 \% \mathrm{~N}, 45 \% \mathrm{P}$ and $18 \% \mathrm{~K}$ were left as remnants (twigs and crown) in the area, with an average equivalent monetary fertilizer (in $\mathrm{R} \$ . \mathrm{ha}^{-1}$ ) ranging from 303.56 to $1,140.78$ depending on the location and site classification of the propriety.
\end{abstract}

Keywords: stand-level models, forest residuals, fertilization. 


\section{INTRODUÇÃO}

A importância da bracatinga, como fonte de matéria-prima energética e para a preservação das florestas naturais, remonta ao início do século 20, quando se desencadeou um esforço para o fomento da espécie como produtora de lenha, estimulando um aumento gradativo da superfície ocupada pelos bracatingais na região metropolitana de Curitiba e demais locais da Região Sul do País (Porfírio da Silva et al., 2006).

Carpazezzi (2006) discorreu que, mesmo decorridos cerca de 100 anos de história dos bracatingais manejados tradicionalmente na região metropolitana de Curitiba, a situação geral dos talhões de cultivo permanece muito parecida. $\mathrm{Ou}$ seja: corte a cada sete anos e regeneração pela ação do fogo, com plantio intercalar de culturas de ciclo curto (principalmente milho e feijão) entre tocos, nos seis meses iniciais. Os tratos culturais continuam poucos, a reposição de nutrientes por adubação é ínfima quando existente, é restrita às culturas agrícolas, sem calagem ou orientação técnica - e nenhum material genético apropriado para o sistema foi desenvolvido.

Para a perpetuação das florestas de bracatinga, bem como das atividades econômicas ligadas à espécie, estudos sobre a quantificação da biomassa vegetal e dos nutrientes minerais presentes, além da modelagem estatística dos mesmos, se tornam fundamentais para o uso responsável dos recursos florestais. Diversos trabalhos foram desenvolvidos com o intuito de modelagem e quantificação dos nutrientes em componentes da biomassa florestal (Bellote et al., 1980; Baggio \& Carpanezzi, 1997b; Schumacher \& Caldeira, 2001; Caldeira et al., 2003; Schumacher et al., 2008; Téo et al., 2010) para embasar as atividades silviculturais e a devida reposição de nutrientes.

Santana et al. (1999) afirmaram que em solos com baixos teores de nutrientes em formas disponíveis e totais, como é o caso dos solos dos bracatingais, a exportação de elevadas quantidades de nutrientes pela colheita florestal ao longo das rotações pode levar à redução da capacidade produtiva do sítio; daí, a importância da identificação de locais pela produtividade e pela fertilidade do solo. Baggio \& Carpanezzi (1997a) asseguraram que o conhecimento do conteúdo de nutrientes dos resíduos é importante no sentido de determinar seu valor econômico e buscar alternativas para manter os nutrientes no terreno.

Dessa forma, o presente trabalho objetivou quantificar o conteúdo de macronutrientes presentes nos compartimentos da biomassa aérea dos bracatingais nativos, localizados em diferentes municípios da região metropolitana de Curitiba. Para tanto, utilizaram-se modelos de povoamento para uma posterior mensuração dos benefícios econômicos proporcionados pelos resíduos florestais mantidos na área após a exploração.

\section{MATERIAL E MÉTODOS}

\subsection{Caracterização do estudo}

Os dados dendrométricos das 304 parcelas temporárias instaladas em bracatingais nativos foram coletados em 15 municípios da região metropolitana de Curitiba (Figura 1). A unidade fitogeográfica dos bracatingais é caracterizada como faixas transicionais entre a Floresta Ombrófila Densa e a Floresta Ombrófila Mista, com dominância de Araucaria angustifolia. A geologia da região é de origem metamórfica, resultando numa topografia fortemente ondulada, com solos muito heterogêneos no que se refere à profundidade e à fertilidade $\mathrm{O}$ clima, de acordo com a classificação de Köeppen, é o $\mathrm{Cfb}$, com temperatura média anual de $16,5^{\circ} \mathrm{C}$.

Os bracatingais estão inseridos em solos dos tipos: Latossolo Roxo Álico e Distrófico, Latossolo Vermelho-Escuro Álico e Distrófico, Latossolo Vermelho-Amarelo Álico pouco profundo, Podzólico Vermelho-Amarelo Álico, Cambissolo Álico e Distrófico, e Solos LitólicosÁlicos e Distróficos (Embrapa, 1984).

\subsection{Estimativa do conteúdo de macronutrientes}

As equações utilizadas para estimativa do conteúdo de nutrientes presentes nos diferentes compartimentos da biomassa aérea dos bracatingais (Tabela 1) foram desenvolvidas em estudo realizado por Ribeiro et al. (2012), em que: $\mathrm{Ln}=$ logaritmo 


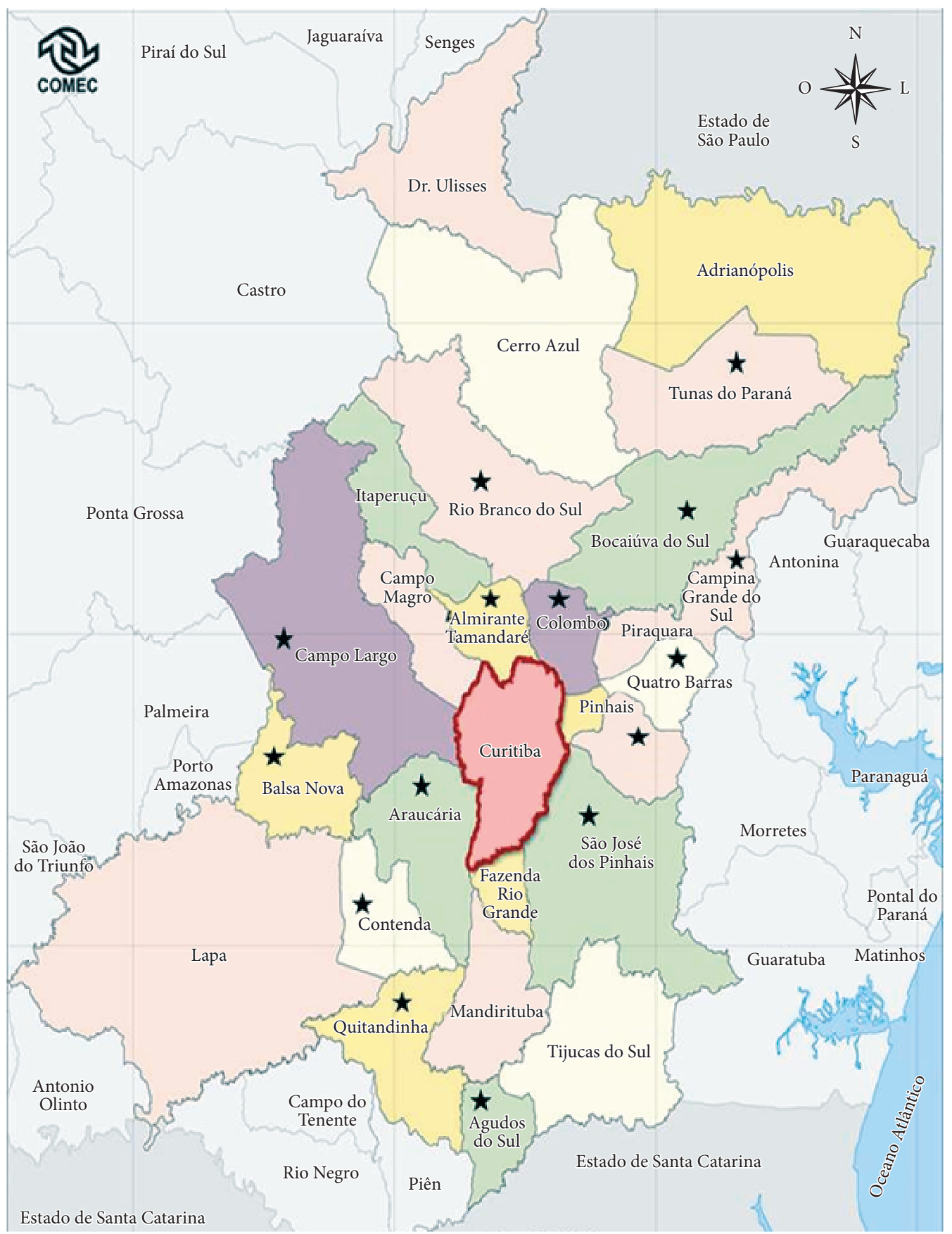

Figura 1. Região metropolitana de Curitiba, com destaque ( $\star$ ) para os municípios de onde os dados foram coletados (Fonte: Paraná, 2009).

Figure 1. Curitiba metropolitan region pointing out $(\star)$ counties where data came from.

natural; $\mathrm{G}=$ área basal $\left(\mathrm{m}^{2} \cdot \mathrm{ha}^{-1}\right) ; \mathrm{h}=$ altura média $(\mathrm{m})$; hd = altura dominante média $(\mathrm{m}) ; \mathrm{I}=$ idade (anos), e IS = classe de sítio $(\mathrm{m})$. Os autores citados, após testarem diferentes modelos matemáticos, optaram pelos modelos com melhores estatísticas de ajuste e precisão, e distribuição homogênea dos resíduos para a quantificação dos macronutrientes.

A 'copa' foi considerada como sendo os galhos com diâmetro inferior a $4 \mathrm{~cm}$, as folhas e os galhos mortos; já o compartimento 'lenha' foi definido 
como sendo o peso seco dos componentes fuste, casca e galhos, com diâmetro maior ou igual a $4 \mathrm{~cm}$, e a biomassa aérea total foi considerada como a soma dos dois compartimentos.

\subsection{Valoração econômica dos macronutrientes}

A quantificação dos ganhos potenciais de macronutrientes foi feita por meio do seu peso equivalente em fertilizantes comerciais, conforme Baggio \& Carpanezzi (1997a, b).

As concentrações dos nutrientes nas formulações foram fornecidas pelos fabricantes e os elementos que se repetiram na formulação química foram devidamente subtraídos dos totais.

Os valores dos fertilizantes foram baseados nas cotações do mês de novembro de 2011, realizadas pela Secretaria da Agricultura e Abastecimento do
Estado do Paraná. Todos os dados para o cálculo do benefício econômico dos nutrientes na porção da biomassa remanescente nos bracatingais após a exploração estão listados na Tabela 2.

Os respectivos preços de nutrientes, correspondentes ao ano de 2011, foram multiplicados pelo conteúdo médio estimado de cada macronutriente. Dessa forma, obteve-se, naquele período, o benefício econômico proporcionado pelos nutrientes da biomassa aérea de povoamentos de bracatinga equivalente à fertilização química.

\section{RESULTADOS E DISCUSSÃO}

As estatísticas descritivas das principais variáveis coletadas nas 304 parcelas estão listadas na Tabela 3. Nota-se que a maior variação ocorreu no número de indivíduos, na idade e na área basal; tal fato é

Tabela 1. Equações selecionadas para estimativa dos macronutrientes em povoamentos de bracatinga e suas respectivas estatísticas de ajuste e precisão.

Table 1. Selected equations for estimating macronutrients in bracatinga stands and their respective statistics.

\begin{tabular}{|c|c|c|c|}
\hline Biomassa & Modelo & $S_{y x}(\%)$ & $\mathbf{R}^{2}{ }_{\mathrm{aj}}(\%)$ \\
\hline Total & $\operatorname{Ln}(\mathrm{Ca})=1,41233+0,49573^{\star} \operatorname{Ln}\left(\mathrm{G}^{2} \cdot \mathrm{h}\right)$ & 5,65 & 99,33 \\
\hline Lenha & $\operatorname{Ln}(\mathrm{Ca})=1,03497+0,99333^{\star} \operatorname{Ln}(\mathrm{G})+0,52348^{\star} \operatorname{Ln}(\mathrm{h})$ & 18,63 & 87,41 \\
\hline Copa & $\operatorname{Ln}(\mathrm{Ca})=1,25125+1,01221^{\star} \operatorname{Ln}(\mathrm{G})$ & 15,49 & 89,93 \\
\hline Total & $\operatorname{Ln}(\mathrm{Mg})=-2,11911+0,71729^{*} \operatorname{Ln}(\mathrm{G} \cdot \mathrm{h})+0,28278^{*} \operatorname{Ln}(\mathrm{G} . \mathrm{hd})$ & 2,67 & 99,69 \\
\hline Lenha & $\mathrm{Mg}=0,05719+0,0579052^{\star}(\mathrm{G} . \mathrm{h})+0,0233453^{\star}(\mathrm{G} . \mathrm{hd})$ & 2,55 & 99,72 \\
\hline Copa & $\mathrm{Mg}=0,0276019+0,0271591^{\star}(\mathrm{G} . \mathrm{h})+0,0109649^{*}(\mathrm{G} . \mathrm{hd})$ & 2,56 & 99,72 \\
\hline Total & $\operatorname{Ln}(\mathrm{N})=2,12010+0,49370^{*}\left(\mathrm{G}^{2} \cdot \mathrm{h}\right)$ & 15,10 & 91,27 \\
\hline Lenha & $\operatorname{Ln}(N)=1,63719+0,49368^{\star}\left(G^{2} \cdot h\right)$ & 15,27 & 91,60 \\
\hline Copa & $\operatorname{Ln}(N)=2,34832+1,00197^{\star} \operatorname{Ln}(G)$ & 17,69 & 87,54 \\
\hline Total & $\operatorname{Ln}(P)=-0,83821+0,99626^{\star} \operatorname{Ln}(G)+0,29487^{\star} \operatorname{Ln}(h)$ & 14,43 & 91,68 \\
\hline Lenha & $\operatorname{Ln}(P)=-1,42724+0,99402^{\star} \operatorname{Ln}(G)+0,29293^{\star} \operatorname{Ln}(h)$ & 12,95 & 93,20 \\
\hline Copa & $\operatorname{Ln}(P)=-1,64786+0,99893^{\star} \operatorname{Ln}(G)+0,29726^{\star} \operatorname{Ln}(h)$ & 16,22 & 89,69 \\
\hline Total & $\operatorname{Ln}(K)=1,35817+0,49577^{\star}\left(G^{2} \cdot h\right)$ & 16,89 & 89,58 \\
\hline Lenha & $\operatorname{Ln}(K)=2,47532+0,00826^{\star} I S-2,70034^{\star} I^{-1}+1,05941^{\star} \operatorname{Ln}(G)$ & 16,47 & 90,23 \\
\hline Copa & $\operatorname{Ln}(K)=0,91169+0,98866^{\star} \operatorname{Ln}(G)$ & 20,47 & 84,34 \\
\hline
\end{tabular}

Tabela 2. Características e preços dos fertilizantes adotados para as estimativas de equivalência dos nutrientes.

Table 2. Characteristics and prices of fertilizers adopted to estimate of nutrient equivalency.

\begin{tabular}{ccccc} 
Nutriente & Fertilizante & $\begin{array}{c}\text { Porcentagem do } \\
\text { nutriente }\end{array}$ & $\begin{array}{c}\text { Preço do adubo } \\
\left(\text { reais. } \mathbf{k g}^{-1}\right)\end{array}$ & $\begin{array}{c}\text { Preço do nutriente } \\
\left(\mathbf{r e a i s .}_{\mathbf{k g}} \mathbf{k g}^{-1}\right)\end{array}$ \\
\hline $\mathrm{N}$ & Ureia & 45 & 1,31 & 2,90 \\
$\mathrm{P}$ & Superfosfato simples & $18(\mathrm{P} 2 \mathrm{O} 5)$ & 0,77 & 4,28 \\
$\mathrm{~K}$ & Cloreto de potássio & $60(\mathrm{~K} 2 \mathrm{O})$ & 1,31 & 2,18 \\
$\mathrm{Ca}$ & Calcário dolomítico & $29(\mathrm{CaO})$ & 0,07 & 0,24 \\
$\mathrm{Mg}$ & Calcário dolomítico & $19(\mathrm{MgO})$ & 0,07 & 0,37 \\
\hline
\end{tabular}


explicado pela variabilidade na idade das parcelas e a grande influência dessa variável no ciclo de vida do bracatingal. Por ser uma espécie pioneira e heliófila, a bracatinga apresenta mortalidade acentuada até os cinco anos de idade, com a densidade do povoamento variando de 10 a 40 mil plantas.ha ${ }^{-1}$ no início do ciclo de vida, finalizando o mesmo com 1400 a 3000 mil plantas.ha $^{-1}$ (Machado et al., 2001).

Uma avaliação do conteúdo médio de macronutrientes em todas as regiões em que foram instaladas as parcelas do estudo foi realizada a partir da estimativa dos macronutrientes, valendo-se das equações selecionadas para os três índices de sítio do estudo, uma vez que é esperado um maior conteúdo de nutrientes em sítios mais produtivos. Na Tabela 4, estão listados os valores médios de macronutrientes presentes na biomassa da aérea total e na biomassa da copa; para quantificação dos valores do compartimento 'lenha', o qual foi removido do sítio durante a exploração da madeira, basta efetuar-se a subtração entre os valores totais pelos valores de copas.

Os resultados da Tabela 4 mostram que os municípios de Araucária, Agudos do Sul e Quitandinha são os que apresentaram maior conteúdo médio estimado de macronutrientes. Em contrapartida, as menores quantidades médias de macronutrientes foram estimadas para Tunas do Paraná, Bocaiuva do Sul e Campina Grande do Sul. Nota-se que apenas cinco municípios (Almirante Tamandaré, Bocaiuva do Sul, Campina Grande do Sul, Colombo e Tunas do Paraná) dispunham de parcelas em todos os índices de sítio; os demais municípios se limitaram a conter apenas uma ou duas classes de sítio, dificultando, assim, a comparação de produtividade nos diferentes índices de sítio.
Numa média geral, após a exploração da lenha, restaram, como remanescentes nas áreas, 32\% de Ca, $26 \%$ de $\mathrm{Mg}, 39 \%$ de N, $34 \%$ de $\mathrm{P}$ e $19 \%$ de $\mathrm{K}$, provenientes do compartimento 'copa'. Vieira et al. (2011), avaliando o sistema de exploração de Pinus taeda (madeira + casca) aos nove anos de idade, constataram uma remoção de $41,1 \%$ de Ca; $46,2 \%$ de $\mathrm{Mg} ; 45,9 \%$ de K; $45,4 \%$ de P e 40,0\% de $\mathrm{N}$ dos macronutrientes presentes na biomassa total. Baggio \& Carpanezzi (1997b), ao estudarem bracatingais nativos com sete anos de idade, relataram valores semelhantes aos do presente estudo, sendo 20,3\% de Ca, $28,6 \%$ de $\mathrm{Mg}, 35,1 \%$ de N, $44,6 \%$ de $\mathrm{P}$ e $21,2 \%$ de $\mathrm{K}$ mantidos na área após a exploração (copa).

$\mathrm{Na}$ Figura 2, estão apresentados os benefícios/ ganhos econômicos (reais.ha ${ }^{-1}$ ) dos macronutrientes nos diferentes municípios da região metropolitana de Curitiba, sem a distinção de classes de sítio em cada município. As estimativas foram obtidas caso a exploração das árvores fosse realizada por completo, ou seja, sem a permanência dos resíduos florestais no solo.

Analisando-se a Figura 2, verifica-se que propriedades localizadas no município de Quitandinha terão maior gasto monetário caso a colheita da árvore seja completa, variando de $\mathrm{R} \$ 4.495,71$ por hectare para reposição de nitrogênio a $\mathrm{R} \$ 31,39$ por hectare para reposição do magnésio. Já os bracatingais localizados no município de Tunas do Paraná, possuem valores baixos de reposição de nutrientes, variando de $\mathrm{R} \$ 762,20$ por hectare para nitrogênio a $R \$ 5,37$ por hectare para magnésio. É válido ressaltar que a árvore de bracatinga incorpora no solo cerca de $200 \mathrm{~kg} \cdot \mathrm{ha}^{-1}$ de nitrogênio durante seu ciclo de vida (Carvalho et al., 2003), sendo então sua reposição completa desnecessária por se tratar de uma leguminosa com alto poder de fixação de $\mathrm{N}$.

Tabela 3. Estatísticas descritivas das principais variáveis independentes analisadas.

Table 3. Descriptive statistics of themainindependent variables.

\begin{tabular}{lccccc}
\multicolumn{1}{c}{ Variável } & Mínimo & Máximo & Média & Desvio padrão & Coef. variação (\%) \\
\hline DAP $(\mathrm{cm})$ & 3,67 & 23,45 & 10,77 & 3,85 & 35,96 \\
$\mathrm{H}(\mathrm{m})$ & 6,91 & 20,18 & 12,43 & 2,65 & 21,32 \\
$\mathrm{G}\left(\mathrm{m}^{2} \cdot \mathrm{ha}^{-1}\right)$ & 2,04 & 56,01 & 13,07 & 6,36 & 47,21 \\
$\mathrm{Hdom}(\mathrm{m})$ & 7,50 & 21,33 & 13,79 & 2,83 & 20,67 \\
$\mathrm{~N}\left(\right.$ ind.ha $\left.^{-1}\right)$ & 67 & 12.439 & 1.997 & 1.930 & 97,55 \\
\hline Idade $($ anos $)$ & 3,00 & 18,00 & 8,89 & 3,64 & 41,43 \\
\hline
\end{tabular}


Tabela 4. Conteúdo médio $\left(\mathrm{kg} \cdot \mathrm{ha}^{-1}\right)$ de macronutrientes nos componentes da biomassa aérea total (t) e copa (c) de povoamentos de bracatinga nos diferentes municípios, e índices de sítio (IS).

Table 4. Average macronutrients content in the compartments of total above ground and crown biomass of bracatinga stands in different counties and site indexes.

\begin{tabular}{|c|c|c|c|c|c|c|c|c|c|c|c|}
\hline \multirow{2}{*}{ IS (m) } & \multirow{2}{*}{ Local } & \multicolumn{10}{|c|}{ Conteúdo médio de macronutriente $\left(\mathrm{kg} \cdot \mathrm{ha}^{-1}\right)$} \\
\hline & & $\mathbf{N}(\mathbf{t})$ & $\mathbf{N}(\mathbf{c})$ & $\mathbf{P}(\mathrm{t})$ & $\mathbf{P}(\mathrm{c})$ & $K(t)$ & $\mathrm{K}(\mathrm{c})$ & $\mathrm{Ca}(\mathrm{t})$ & $\mathrm{Ca}(\mathrm{c})$ & $\operatorname{Mg}(t)$ & $\operatorname{Mg}(c)$ \\
\hline \multirow{9}{*}{9} & Almirante Tamandaré & 244,0 & 96,0 & 8,0 & 3,6 & 116,0 & 22,3 & 123,4 & 32,6 & 12,0 & 3,8 \\
\hline & Bocaiúva do Sul & 210,2 & 88,9 & 7,1 & 3,2 & 99,9 & 20,7 & 108,0 & 30,2 & 10,2 & 3,2 \\
\hline & Campina Grande do Sul & 238,0 & 91,3 & 7,7 & 3,5 & 113,1 & 21,2 & 119,5 & 31,0 & 12,5 & 4,0 \\
\hline & Campo Largo & 347,4 & 130,8 & 11,2 & 5,0 & 165,4 & 30,3 & 174,1 & 44,6 & 18,4 & 5,9 \\
\hline & Colombo & 245,5 & 105,5 & 8,3 & 3,8 & 116,7 & 24,5 & 126,8 & 35,9 & 11,5 & 3,7 \\
\hline & Piraquara & 337,6 & 164,6 & 12,0 & 5,4 & 160,6 & 38,0 & 180,0 & 56,2 & 14,6 & 4,7 \\
\hline & Rio Branco do Sul & 328,5 & 133,4 & 10,9 & 4,9 & 156,3 & 30,9 & 167,5 & 45,5 & 15,9 & 5,1 \\
\hline & São José dos Pinhais & 705,9 & 319,5 & 24,5 & 11,1 & 337,0 & 73,1 & 371,6 & 109,9 & 32,4 & 10,3 \\
\hline & Tunas do Paraná & 207,0 & 82,8 & 6,8 & 3,1 & 98,3 & 19,3 & 104,9 & 28,1 & 10,2 & 3,3 \\
\hline \multirow{13}{*}{13} & Agudos do Sul & 1122,7 & 459,4 & 59,3 & 18,9 & 537,0 & 104,6 & 37,4 & 17,0 & 578,5 & 158,6 \\
\hline & Almirante Tamandaré & 308,9 & 111,3 & 17,6 & 5,6 & 147,0 & 25,8 & 9,7 & 4,4 & 153,1 & 37,9 \\
\hline & Balsa Nova & 523,6 & 214,7 & 26,6 & 8,5 & 249,7 & 49,4 & 17,4 & 7,9 & 268,5 & 73,5 \\
\hline & Bocaiuva do Sul & 342,8 & 135,4 & 18,1 & 5,8 & 163,2 & 31,3 & 11,2 & 5,1 & 173,7 & 46,2 \\
\hline & Campina Grande do Sul & 356,0 & 123,8 & 21,1 & 6,7 & 169,5 & 28,7 & 11,1 & 5,0 & 175,1 & 42,2 \\
\hline & Campo Largo & 454,1 & 178,0 & 24,3 & 7,8 & 216,4 & 41,0 & 14,8 & 6,7 & 230,1 & 60,9 \\
\hline & Colombo & 339,0 & 125,7 & 18,8 & 6,0 & 161,4 & 29,1 & 10,8 & 4,9 & 169,2 & 42,9 \\
\hline & Piraquara & 513,6 & 207,8 & 26,9 & 8,6 & 244,8 & 47,8 & 17,0 & 7,7 & 262,6 & 71,2 \\
\hline & Quatro Barras & 533,0 & 208,6 & 27,7 & 8,8 & 254,1 & 48,0 & 17,4 & 7,9 & 270,4 & 71,4 \\
\hline & Quitandinha & 1549,6 & 601,3 & 85,2 & 27,2 & 742,1 & 136,4 & 50,6 & 23,0 & 790,0 & 208,2 \\
\hline & Rio Branco do Sul & 335,1 & 125,6 & 18,2 & 5,8 & 159,5 & 29,1 & 10,7 & 4,9 & 167,7 & 42,8 \\
\hline & São José dos Pinhais & 447,6 & 204,1 & 20,4 & 6,5 & 213,3 & 47,0 & 15,5 & 7,0 & 235,3 & 69,9 \\
\hline & Tunas do Paraná & 265,8 & 97,4 & 14,7 & 4,7 & 126,4 & 22,6 & 8,4 & 3,8 & 132,1 & 33,1 \\
\hline \multirow{10}{*}{17} & Almirante Tamandaré & 765,8 & 311,9 & 40,7 & 13,0 & 365,7 & 71,4 & 25,4 & 11,5 & 393,2 & 107,3 \\
\hline & Araucária & 1040,0 & 417,9 & 57,0 & 18,2 & 497,2 & 95,3 & 34,4 & 15,6 & 533,3 & 144,1 \\
\hline & Balsa Nova & 734,3 & 290,8 & 41,0 & 13,1 & 350,6 & 66,6 & 24,1 & 10,9 & 374,4 & 99,9 \\
\hline & Bocaiuva do Sul & 432,6 & 161,1 & 24,9 & 7,9 & 206,1 & 37,2 & 13,8 & 6,3 & 216,4 & 55,1 \\
\hline & Campina Grande do Sul & 492,6 & 152,4 & 32,8 & 10,5 & 234,8 & 35,2 & 14,6 & 6,6 & 236,0 & 52,0 \\
\hline & Colombo & 335,2 & 120,7 & 19,4 & 6,2 & 159,5 & 28,0 & 10,5 & 4,8 & 166,0 & 41,1 \\
\hline & Piraquara & 512,9 & 189,3 & 29,1 & 9,3 & 244,5 & 43,6 & 16,3 & 7,4 & 256,5 & 64,8 \\
\hline & Quatro Barras & 607,3 & 239,7 & 32,2 & 10,3 & 289,7 & 55,1 & 19,9 & 9,0 & 309,0 & 82,2 \\
\hline & Tunas do Paraná & 340,3 & 110,2 & 21,7 & 6,9 & 162,0 & 25,6 & 10,3 & 4,6 & 164,4 & 37,5 \\
\hline & Media geral & 490,9 & 193,5 & 25,0 & 8,4 & 234,1 & 44,5 & 60,6 & 19,2 & 206,2 & 54,4 \\
\hline
\end{tabular}

Como dito anteriormente, apenas cinco municípios se enquadraram em todas as classes de sítio. Portanto, apenas para estes, os valores econômicos em reais dos resíduos florestais estão apresentados na Tabela 5, cujos resultados foram calculados baseando-se nos valores do quilograma de cada nutriente, conforme descrito na Tabela 2.

A mensuração dos benefícios econômicos dos macronutrientes contidos na biomassa dos resíduos resultou em valores mais altos para o maior índice de sítio, sendo o valor máximo da retirada de nutrientes com a exploração da lenha encontrado no município de Almirante Tamandaré ( $\mathrm{R} \$ 1.140,78$ ) para o IS $=17 \mathrm{~m}$ e o menor valor, para o município de Tunas do Paraná no IS $=9 \mathrm{~m}(\mathrm{R} \$ 303,56)$.

Vieira \& Schumacher (2010) afirmaram que a saída de nutrientes via exportação deve ser um componente levado em consideração na hora do planejamento florestal. Tal preocupação evita que haja diminuição de produtividade com as rotações futuras e também contribui para o manejo sustentável, pois a exportação de nutrientes, principalmente do cálcio, é 


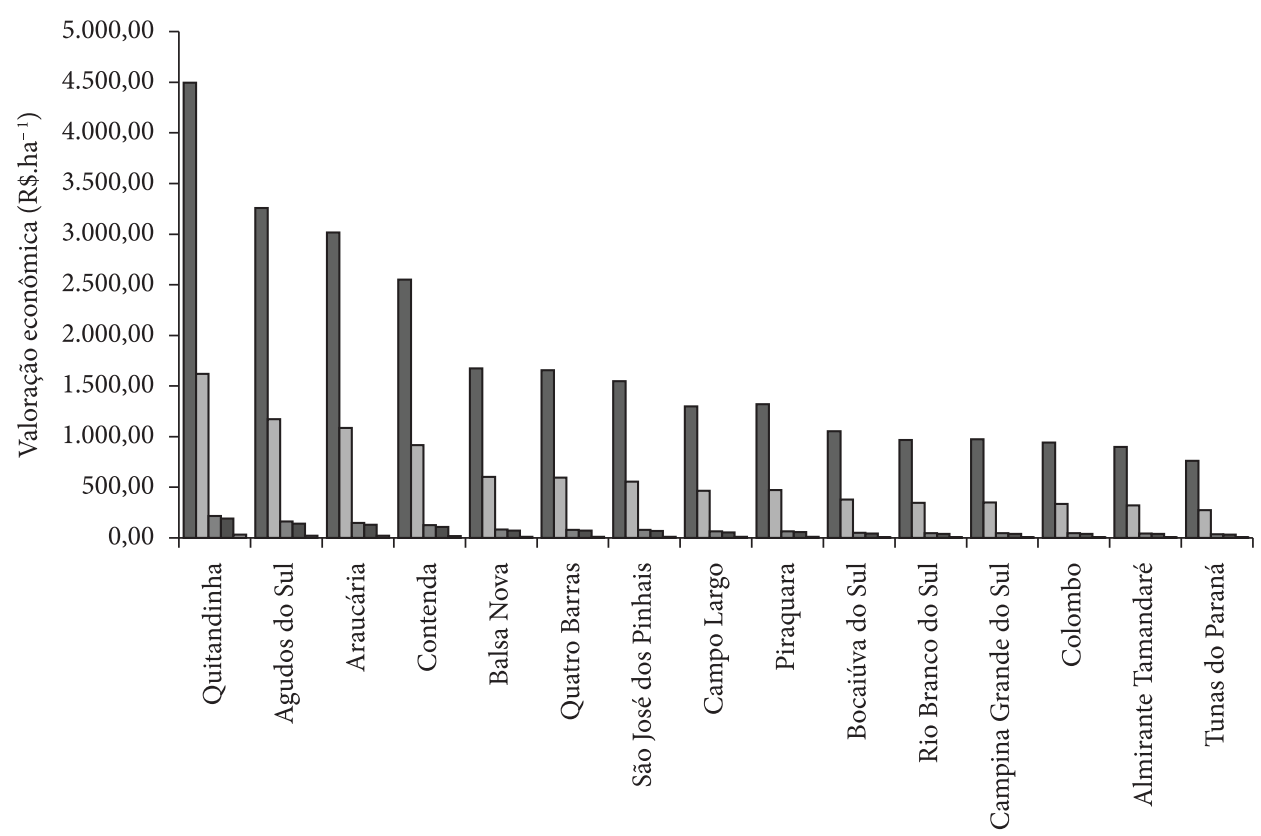

$\square \mathrm{N} \square \mathrm{K} \square \mathrm{P} \quad \square \mathrm{Ca} \quad \square \mathrm{Mg}$

Figura 2. Benefícios econômicos dos macronutrientes presentes na biomassa aérea total de bracatingais nativos nos diferentes municípios da região metropolitana de Curitiba.

Figure 2. Economic benefitsof macronutrients in the total above ground biomass of native bractinga stands in different counties of the Curitiba metropolitan region.

Tabela 5. Valoração econômica (reais.ha ${ }^{-1}$ ) dos macronutrientes contidos nos resíduos florestais em povoamentos de bracatinga em diferentes locais e índices de sítio.

Table 5. Economic valuation (reais.ha ${ }^{-1}$ ) of the macronutrients in the forest residues of bracatinga in different sites and site indexes.

\begin{tabular}{|c|c|c|c|c|c|c|c|}
\hline IS (m) & Local & $\mathbf{N}$ & $\mathbf{P}$ & $\mathbf{K}$ & $\mathrm{Ca}$ & Mg & Total \\
\hline \multirow{5}{*}{9} & Colombo & 306,04 & 16,05 & 53,46 & 8,66 & 1,36 & 385,57 \\
\hline & Almirante Tamandaré & 278,48 & 15,40 & 48,67 & 7,88 & 1,42 & 351,85 \\
\hline & Campina Grande do Sul & 265,03 & 14,86 & 46,39 & 7,49 & 1,47 & 335,23 \\
\hline & Bocaiuva do Sul & 257,97 & 13,64 & 45,16 & 7,29 & 1,20 & 325,25 \\
\hline & Tunas do Paraná & 240,33 & 13,14 & 42,10 & 6,79 & 1,20 & 303,56 \\
\hline \multirow{5}{*}{13} & Bocaiuva do Sul & 392,97 & 21,69 & 68,41 & 11,15 & 2,13 & 496,36 \\
\hline & Colombo & 364,71 & 20,91 & 63,53 & 10,35 & 2,22 & 461,71 \\
\hline & Campina Grande do Sul & 359,24 & 21,41 & 62,57 & 10,19 & 2,48 & 455,90 \\
\hline & Almirante Tamandaré & 322,79 & 18,82 & 56,30 & 9,15 & 2,07 & 409,13 \\
\hline & Tunas do Paraná & 282,54 & 16,30 & 49,38 & 7,99 & 1,73 & 357,95 \\
\hline \multirow{5}{*}{17} & Almirante Tamandaré & 904,91 & 49,31 & 155,88 & 25,89 & 4,79 & $1.140,78$ \\
\hline & Bocaiuva do Sul & 467,33 & 26,74 & 81,15 & 13,29 & 2,93 & 591,44 \\
\hline & Campina Grande do Sul & 442,24 & 28,32 & 76,91 & 12,56 & 3,86 & 563,88 \\
\hline & Colombo & 350,10 & 20,41 & 61,05 & 9,92 & 2,28 & 443,76 \\
\hline & Tunas do Paraná & 319,66 & 19,88 & 55,83 & 9,05 & 2,55 & 406,97 \\
\hline
\end{tabular}

elevado em função da colheita da madeira com casca. Nesse sentido, deve-se evitar a remoção dos resíduos (folhas, cascas e galhos) durante a colheita florestal, contribuindo com a sustentabilidade ambiental por meio do incremento da disponibilidade de nutrientes após a decomposição desse material e, consequentemente, diminuindo o uso de adubação química para reposição nutricional do sítio florestal. 
Novamente, Baggio et al. (1986) já ressaltavam que a bracatinga, por ser leguminosa, tem a capacidade de amenizar danos ambientais, mesmo com a aplicação do fogo para quebra de dormência no banco de sementes; sendo assim, os valores de reposição para nitrogênio devem ser revistos para não ocorrer uma superdosagem no solo.

Baggio \& Carpanezzi (1997a), realizando um estudo semelhante a este trabalho, concluíram que a exportação de nutrientes pela lenha da bracatinga representa ao menos $10,3 \%$ da renda bruta obtida por sua produção, sendo a reposição dos fertilizantes inviável do ponto de vista econômico. Decorre, então, a necessidade de introduzir mudanças profundas nas práticas do sistema no sentido de equilibrar o balanço de nutrientes, fato ainda corriqueiro nos dias de hoje. Os valores potenciais dos nutrientes, contidos nos resíduos e exportados em razão da queima, implicam em custos que devem ser considerados nas estimativas de rentabilidade dos sistemas tradicionais da bracatinga (Baggio \& Carpanezzi, 1997b).

\section{CONCLUSÕES}

Em ordem decrescente, os valores estimados de macronutrientes encontrados na parte aérea dos bracatingais configuram-se na sequência de $\mathrm{N}>\mathrm{Ca}>\mathrm{K}>\mathrm{Mg}>\mathrm{P}$.

A avaliação econômica dos componentes da biomassa aérea dos povoamentos de bracatinga indica que, caso a colheita da árvore seja completa, o produtor deverá desembolsar valores monetários para reposição dos macronutrientes, variando de $\mathrm{R} \$ 1.107,45$ a $\mathrm{R} \$ 6.554,47$ por hectare, dependendo do local da propriedade e da classificação de sítio, desconsiderando-se a incorporação natural de nutrientes nos solos dos bracatingais.

Portanto, a manutenção dos resíduos florestais no solo após o processo de exploração é fundamental para que ocorra a reincorporação dos nutrientes de forma natural, além do montante que pode ser poupado pelo produtor ( $\mathrm{R} \$ 303,56$ a $1.140,78$ por hectare), dispensando parte do uso de fertilizantes químicos e uso de maquinário para aplicação no solo.

\section{STATUS DA SUBMISSÃO}

Recebido: 20/03/2012

Aceito: 09/07/2012

Publicado: 31/12/2012

\section{AUTOR(ES) PARA CORRESPONDÊNCIA}

\section{Andressa Ribeiro}

Universidade Federal do Paraná - UFPR, Av. Prefeito Lothário Meissner, 632, Jardim Botânico, CEP 80210-170, Curitiba, PR, Brasil e-mail: andressa.florestal@gmail.com

\section{Sebastião do Amaral Machado}

Universidade Federal do Paraná - UFPR, Av. Prefeito Lothário Meissner, 632, Jardim Botânico, CEP 80210-170, Curitiba, PR, Brasil e-mail: samachado@ufpr.br

\section{REFERENNCIAS}

Baggio AJ, Carpanezzi AA, Graça LR, Ceccon E. Sistema agroflorestal tradicional da bracatinga com culturas agrícolas anuais. Boletim de Pesquisa Florestal 1986; 12: 73-82.

Baggio AJ, Carpanezzi AA. Exportação de nutrientes na exploração de bracatingais. Boletim de Pesquisa Florestal 1997a; 34: 3-15.

Baggio AJ, Carpanezzi AA. Estoque de nutrientes nos resíduos da exploração de bracatingais. Boletim de Pesquisa Florestal 1997b; 34: 17-29.

Bellote AFJ, Sarruge JR, Haag HP, Oliveira GD. Extração e exportação de nutrientes pelo Eucalyptusgrandis Hill ex-Maiden em função da idade: 1- Macronutrientes. Instituto de Pesquisas e Estudos Florestais - IPEF 1980; 20: 1-23.

Caldeira MVW, Rondon Neto RM, Schumacher MV. Conteúdo e exportação de micronutrientes em Acácia-Negra (Acacia mearnsii De Wild.) procedência Batemans Bay (Austrália). Árvore 2003; 27(1): 9-14.

Carpazezzi AA. Aspectos técnicos da produção de bracatinga. In: Porfírio da Silva V, Mazuchowski JZ, Pinto AF, Miniolli Netto J, Becker JC. Memórias da oficina sobre bracatinga no Vale do Ribeira. Embrapa Florestas; 2006. Documentos - Embrapa Florestas.

Carvalho PER, Medrado MJS, Hoeflich VA. Cultivo da bracatinga. Colombo: Embrapa Florestas, 2003. [cited 2012 mar. 12]. Available from: http:// sistemasdeproducao.cnptia.embrapa.br/FontesHTML/ Bracatinga/CultivodaBracatinga/20_equipe.htm. 
Machado SA, Tonon AEN, Oliveira EB, Figueiredo Filho A, Carpanezzi AA. Efeitos da densidade inicial e do sítio sobre o desenvolvimento de bracatingais nativos da região metropolitana de Curitiba. Boletim Pesquisa Florestal 2001; 43: 19-46.

Paraná. Governo do Estado. Secretaria de Estado do Desenvolvimento Urbano. Coordenação da Região Metropolitana de Curitiba - COMEC. Região Metropolitana de Curitiba. COMEC, 2009. [cited 2012 mar. 12]. Available from: http://www.comec.pr.gov.br/ arquivos/File/RMC_2008_politico1.pdf.

Porfírio da Silva V, Mazuchowski JZ, Pinto AF, Miniolli Netto J, Becker JC. Memórias da oficina sobre bracatinga no Vale do Ribeira. Embrapa Florestas; 2006. 109 p. Documentos - Embrapa Florestas.

Ribeiro A, Machado AS, Péllico Netto S, Figueiredo Filho A. Quantificação e modelagem dos macronutrientes em povoamentos de bracatinga. Pesquisa Florestal Brasileira 2012; 32(69): 29-43. http:// dx.doi.org/10.4336/2012.pfb.32.69.29

Santana RC, Barros NF, Neves JCL. Biomassa e conteúdo de nutrientes de procedências de Eucalyptusgrandis e
Eucalyptussaligna em alguns sítios florestais do Estado de São Paulo. Scientia Forestalis 1999; 56: 155-169.

Schumacher MV, Caldeira MVW. Estimativa da biomassa e do conteúdo de nutrientes de um povoamento de Eucalyptusglobulus(Labillardière) subespéciemaidenii. Ciência Florestal 2001; 11(1): 45-53.

Schumacher MV, Brun EJ, Illana VB, Dissiuta SI, Agne TL. Biomassa e nutrientes em um povoamento de HoveniadulcisThunb., plantado na FEPAGRO Florestas, Santa Maria, RS. Ciência Florestal 2008; 18(1): 27-37.

Téo SJ, Machado AS, Figueiredo Filho A, Reissmann CB. Models for estimating macronutrients in Mimosa scabrella Bentham. Cerne 2010; 16(3): 323-334.

Vieira M, Schumacher MV, Bonacina DM. Biomassa e nutrientes removidos no primeiro desbaste de um povoamento de Pinus taeda L. em Cambará do Sul, RS. Árvore 2011; 35(3): 371-379.

Vieira M, Schumacher MV. Exportação de nutrientes pela colheita do Pinus. Caderno de Pesquisa Série Biologia 2010; 22(3): 13-23. 